\title{
Concentrações séricas de zinco e transtornos mentais comuns: Uma revisão
}

\section{integrativa}

\author{
Zinc serum levels and common mental disorders: An integrative review \\ Concentraciones séricas de zinc y trastornos mentales comunes: Una revision integradora
}

Recebido: 25/04/2021 | Revisado: 03/05/2021 | Aceito: 05/05/2021 | Publicado: 20/05/2021

\author{
Sarah de Melo Rocha Cabral \\ ORCID: https://orcid.org/0000-0002-4926-6876 \\ Universidade Federal do Piauí, Brasil \\ E-mail: sarahmelo_9@ @otmail.com \\ Layonne de Sousa Carvalho \\ ORCID: https://orcid.org/0000-0002-7795-035X \\ Universidade Federal do Piauí, Brasil \\ E-mail: layonnesc@hotmail.com \\ Alana Paulina de Moura Sousa \\ ORCID: https://orcid.org/0000-0001-9999-3441 \\ Universidade Federal do Piauí, Brasil \\ E-mail: pmoura2512@gmail.com \\ Marize Melo dos Santos \\ ORCID: https://orcid.org/0000-0003-0699-8062 \\ Universidade Federal do Piauí, Brasil \\ E-mail: marizesantos@ufpi.edu.br
}

\begin{abstract}
Resumo
Objetivou-se analisar a produção científica acerca das evidências atuais sobre a relação entre as concentrações séricas de zinco e transtornos mentais comuns. Trata-se de uma revisão integrativa da literatura, realizada entre março e julho de 2020, utilizando a estratégia PICOS para selecão dos artigos. As bases de dados foram acessadas por meio da Biblioteca Virtual em Saúde e Portal de Periódicos da Capes, correspondendo às: Science Direct, Springer Science, Medline/Pubmed, ProQuest LLC, AGRIS, Bentham Science Publishers, Cengage Learning, Web of Science, John Wiley \& Sons, Scopus, Directory of Open Access Journals, Maney Publishing, e SAGE Publications. Foram analisadas sete publicações que avaliaram indivíduos de ambos os sexos, em diferentes faixas etárias, baseando-se em estudos observacionais analíticos. Os estudos elencados para análise, conduzidos em grupos de diferentes faixas etárias e número amostral, apresentaram resultados semelhantes, demonstrando prevalências de concentrações reduzidas de zinco no sangue de pacientes com depressão e/ou ansiedade. A correlação entre concentrações séricas de zinco e transtornos mentais comuns, na maioria dos resultados, converge para evidências de que a deficiência de zinco sérico está significativamente associada à sintomatologia depressiva e de ansiedade. Baseando-se nos delineamentos das investigações elencadas, os mesmos não podem determinar uma relação de causalidade entre o zinco e os sintomas depressivos e de ansiedade. Porém, é plausível afirmar, com bases nos resultados e mecanismos fisiológicos do zinco, que a baixa concentração periférica deste elemento pode desempenhar um papel na fisiopatologia de algum domínio da função mental.
\end{abstract}

Palavras-chave: Deficiência de zinco; Depressão; Transtornos de ansiedade; Transtornos mentais.

\begin{abstract}
The objective was to analyze the scientific production about the current evidence on the relationship between serum zinc concentrations and common mental disorders. This is an integrative literature review, carried out between March and July 2020, using the PICOS strategy for the selection of articles. The databases were accessed through the Capes Virtual Health Library and Capes Journals Portal, corresponding to: Science Direct, Springer Science, Medline / Pubmed, ProQuest LLC, AGRIS, Bentham Science Publishers, Cengage Learning, Web of Science, John Wiley \& Sons, Scopus, Directory of Open Access Journals, Maney Publishing, and SAGE Publications. Seven publications were analyzed that evaluated individuals of both sexes, in different age groups, based on observational analytical studies. The studies listed for analysis, conducted in groups of different age groups and sample numbers, showed similar results, demonstrating the prevalence of reduced concentrations of zinc in the blood of patients with depression and / or anxiety. The correlation between serum zinc concentrations and common mental disorders, in most results, converges to evidence that serum zinc deficiency is significantly associated with depressive and anxiety symptoms. Based on the designs of the investigations listed, they cannot determine a causal relationship between zinc and depressive and anxiety symptoms. However, it is plausible to state, based on the results and physiological
\end{abstract}


mechanisms of zinc, that the low peripheral concentration of this element may play a role in the pathophysiology of some domain of mental function.

Keywords: Zinc deficiency; Depression; Anxiety; Mental disorders.

\section{Resumen}

El objetivo fue analizar la producción científica sobre la evidencia actual sobre la relación entre las concentraciones séricas de zinc y los trastornos mentales comunes. Se trata de una revisión integradora de la literatura, realizada entre marzo y julio de 2020, utilizando la estrategia PICOS para la selección de artículos. Se accedió a las bases de datos a través de Virtual Health Library y Capes Journals Portal, correspondientes a: Science Direct, Springer Science, Medline / Pubmed, ProQuest LLC, AGRIS, Bentham Science Publishers, Cengage Learning, Web of Science, John Wiley \& Sons, Scopus, Directorio de revistas de acceso abierto, Maney Publishing y SAGE Publications. Se analizaron siete publicaciones que evaluaron a individuos de ambos sexos, en diferentes grupos de edad, con base en estudios analíticos observacionales. Los estudios enumerados para el análisis, realizados en grupos de diferentes grupos de edad y muestras, mostraron resultados similares, lo que demuestra la prevalencia de concentraciones reducidas de zinc en la sangre de pacientes con depresión y / o ansiedad. La correlación entre las concentraciones séricas de zinc y los trastornos mentales comunes, en la mayoría de los resultados, converge en la evidencia de que la deficiencia de zinc sérico se asocia significativamente con síntomas depresivos y de ansiedad. Con base en los diseños de las investigaciones enumeradas, no pueden determinar una relación causal entre el zinc y los síntomas depresivos y de ansiedad. Sin embargo, es plausible afirmar, con base en los resultados y mecanismos fisiológicos del zinc, que la baja concentración periférica de este elemento puede jugar un papel en la fisiopatología de algún dominio de la función mental.

Palabras clave: Deficiencia de zinc; Depresión; Trastornos de ansiedade; Trastornos mentales.

\section{Introdução}

Os transtornos mentais comuns (TMC) referem-se à condições clínicas suficientes para diagnósticos de depressão e ansiedade segundo as classificações do Diagnostic and Statistical Manual of Mental Disorders- $5^{\text {a }}$ edição (DSM-V) e Classificação Internacional de Internacional de Doenças- 11ª revisão (CID-11) (Santos et al., 2019).

A depressão é caracterizada por tristeza, perda de interesse ou prazer, sentimento de culpa ou autoestima baixa, perturbação do sono ou do apetite, sentimentos de cansaço e baixa concentração. Pode ser longa ou recorrente, prejudicando substancialmente a capacidade de um indivíduo lidar com condições rotineiras, sejam na vida pessoal, no trabalho ou na escola. Na forma mais grave, a depressão pode levar ao suicídio (World Health Organization \& Organização Pan-Americana da Saúde, 2020).

Os distúrbios de ansiedade são caracterizados por sentimentos de ansiedade e medo, incluindo transtorno de ansiedade generalizada, transtorno de pânico, fobias, transtorno de ansiedade social, transtorno obsessivo-compulsivo e transtorno de estresse pós-traumático. Tal como acontece com a depressão, os sintomas podem variar de leve a grave (World Health Organization, 2017; World Health Organization, 2020b).

A Organização Mundial da Saúde (OMS) estima que 4,4\% da população global sofre de transtorno depressivo e 3,6\% de transtorno de ansiedade. No Brasil, a prevalência é de 5,8\% e 9,3\% da população, respectivamente (World Health Organization, 2017).

Fatores sociodemográficos como idade adulta, sexo feminino, raça negra, estado civil divorciado ou solteiro, baixa renda e escolaridade, bem como fatores ambientais, nutricionais e conflitos familiares estão associados à maior ocorrência dos TMC (Silva et al., 2019; Henrique et al., 2018; Senicato et al., 2018).

Diante das atuais barreiras para o tratamento efetivo desses transtornos, estudiosos estão buscando alternativas no campo da nutrição para melhor entender e abordar questões em saúde mental (Kaner et al., 2015; Li at al., 2020; Nguyen et al., 2019). Dados clínicos e epidemiológicos concentram-se na importância do estado nutricional do zinco em todos os sistemas fisiológicos, incluindo o funcionamento neural, onde participa de muitos processos celulares (Joe et al., 2018; Campo et al., 2016).

No organismo, a quantidade total de zinco é de $1,5 \mathrm{~g}$ a 2,5g, distribuindo-se por todas as estruturas, principalmente nos 
músculos estriados (60\%), ossos (20 a 30\%) e fígado (4 a 6\%). Apenas 0,1\% do zinco total encontra-se na corrente sanguínea e, desses, $90 \%$ estão nos eritrócitos, $9 \%$ no plasma e $1 \%$ nos leucócitos. A concentração plasmática de zinco varia entre 70 e $120 \mathrm{mcg} / \mathrm{dL}$, sendo $60 \%$ ligados à albumina e 30\% ligados à macroglobulina (Ciampo \& Ciampo, 2014).

A conexão entre a desregulação do zinco (tanto ingestão dietética como concentrações sanguíneas insuficientes) e as doenças psiquiátricas está sendo continuamente esclarecida. Descobertas clínicas, moleculares e genéticas convergem para a associação da homeostase deste elemento com diagnóstico de depressão clínica e psicose (Markiewic-Żukowska et al., 2015; Petrilli et al., 2017), relação esta que está sendo bem apreciada na interface clínica.

Alguns mecanismos podem explicar a ação do zinco na prevenção de transtornos psiquiátricos. Um deles pode estar ligado à ação do zinco no fator neurotrópico derivado do cérebro (BDNF), um fator de crescimento que promove neurogênese e diferenciação. O zinco interage com os níveis de BDNF e sua deficiência diminui a neurogênese e os sintomas depressivos se manifestam (Youssef et al., 2018).

Consideração adicional da ação do zinco com o BDNF é a sua interação com os receptores GPR39, receptor de Zn+2 que ativa diversas vias neuronais envolvidas na resposta adaptativa ao estresse (Młyniec et al., 2015). Además, o GPR39 provavelmente serve como um elo crucial na interação entre o zinco e o sistema serotoninérgico, necessário para a atividade dos antidepressivos direcionados à via da serotonina (Doboszewska et al., 2017).

A regulação negativa do receptor cortical GPR39 e do nível de proteína BDNF e a gravidade da depressão foi associada à deficiência de zinco (Petrilli et al., 2017; Młyniec et al., 2015).

Outro possível e importante papel do zinco é na sinaptogêne, onde participa na transativação do Receptor de Tirosina Quinase B (TrkB). O TrkB é um fator crucial, cuja ativação é independente da ativação do BDNF e parece ser responsável pela potencial produção de fibras musgosas do hipocampo (Manosso, 2016). Assim, o zinco regula a plasticidade sináptica, auxiliando a neurogênese e prevenindo estados patológicos.

Associado à depressão, a deficiência de zinco pode ser responsável por maior impacto na sintomatologia psiquiátrica (Manosso, 2019; Saueressig et al., 2016). Na atualidade, o transtorno depressivo é causa de morbidade e mortalidade significativas (Mlyniec, 2015; World Health Organization, 2020a; World Health Organization, 2015).

A deficiência intracelular de zinco pode ser causada por absorção prejudicada, condições clínicas, incluindo alcoolismo, e, em especial, insuficiência alimentar (Petrilli et al., 2017).

O objetivo deste artigo foi analisar a produção científica acerca das evidências atuais sobre a relação entre as concentrações séricas de zinco e transtornos mentais comuns.

\section{Metodologia}

Trata-se de um estudo descritivo, exploratório, do tipo revisão integrativa, desenvolvido com base nas seguintes etapas: 1) formulação da pergunta norteadora (problema); 2) busca ou amostragem na literatura; 3) coleta de dados; 4) análise crítica dos estudos incluídos; 5) discussão dos resultados; e 6) apresentação da revisão integrativa (Souza et al., 2010).

O problema formulado (etapa 1) correspondeu à necessidade de se obterem os conhecimentos disponíveis sobre a relação entre as concentrações séricas de zinco e transtornos mentais comuns.

A pergunta de pesquisa foi baseada nos seguintes componentes: 1 (P) Pacientes/População: indivíduos com sintomas de depressão e ansiedade. A idade dos pacientes estudados não foi definida; 2(I) Intervenção: diagnóstica, ou seja, ferramentas utilizadas para dosarem as concentrações séricas de zinco e sintomatologia depressiva e/ou ansiolítica; 3(C) Comparação ou controle: grupos controle ou sem comparação; 4(D) Desfecho: associação entre concentrações séricas de zinco com transtornos mentais comuns; 5 (S) Study type (tipos de estudo): artigos oriundos de estudos observacionais analíticos.

A busca da literatura (etapa 2) foi realizada entre março e julho de 2020. As bases de dados foram acessadas por meio 
da Biblioteca Virtual em Saúde (BVS) e Portal de Periódicos da Capes, correspondendo às seguintes: Science Direct, Springer Science, Medline/Pubmed, ProQuest LLC, AGRIS, Bentham Science Publishers, Cengage Learning, Web of Science, John Wiley \& Sons, Scopus, Directory of Open Access Journals, Maney Publishing, e SAGE Publications.

A estratégia de busca foi feita com os seguintes descritores e operadores booleanos: zinc OR zinc deficiency AND depression OR mental disorders OR anxiety OR mood disorders.

Para a busca e elegibilidade dos artigos foram considerados os seguintes critérios de inclusão: artigos revisados por pares; publicações dos últimos 10 anos (2010 a 2020) nos idiomas inglês, português e espanhol; publicações na íntegra e disponíveis eletronicamente. Foram excluídos os artigos de pesquisa bibliográfica e de reflexão, experimentais, relato de caso, série de casos, teses, dissertações; estudos que envolveram participantes com outros diagnósticos psiquiátricos; pesquisas que não foram realizadas em seres humanos; e aqueles repetidos em diferentes bases de dados.

A etapa de coleta de dados (etapa 3) foi realizada por dois revisores e baseou-se na leitura dos manuscritos que atenderam aos critérios de inclusão na íntegra. Discordâncias foram resolvidas por discussão entre os revisores.

Para extração dos dados dos artigos selecionados, foi utilizado um instrumento, validado por Ursi (2005), contendo informações como identificação do título do artigo, autores, país, idioma e ano de publicação; instituição sede do estudo; tipo de publicação; características metodológicas do estudo como: questão de investigação, amostra, tratamento de dados, intervenção realizada, principais resultados, análises, nível de evidência; e avaliação do rigor metodológico, identificando-se a clareza metodológica, limitações e vieses do estudo.

Os resultados foram sintetizados e os informes chave de cada artigo foram pontuados para análise (etapas 4). Além disso, os manuscritos foram classificados de acordo com o nível de evidência, respeitando-se a hierarquia segundo delineamento da pesquisa (Ursi, 2005): Nível 1 - evidências resultantes da meta-análise de múltiplos estudos clínicos controlados e randomizados; Nível 2 - evidências obtidas em estudos individuais com delineamento experimental; Nível 3 evidências de estudos quase-experimentais; Nível 4 - evidências de estudos descritivos (não-experimentais) ou com abordagem qualitativa; Nível 5 - evidências provenientes de relatos de caso ou de experiência; Nível 6 - evidências baseadas em opiniões de especialistas.

Para apresentação, os resultados foram expostos em quadros, mostrando a caracterização dos estudos, principais achados e limitações. Em seguida, a partir da interpretação, comparou-se os dados evidenciados nas análises ao referencial teórico, bem como foram pontuadas as convergências e divergências entre eles (etapa 5).

\section{Resultados}

Os manuscritos identificados na busca eletrônica foram publicados na língua portuguesa ( $n=01)$ e os demais na língua inglesa (n=97). Após leitura na íntegra, não foram selecionados os estudos que investigaram associação das concentrações séricas de zinco com efeitos de terapia medicamentosa em indivíduos com TMC, bem como análises comparativas das razões de níveis zinco sérico com outros elementos em pacientes com depressão, ansiedade generalizada e/ou outros diagnósticos psiquiátricos. Os resultados da busca, de acordo com etapas de seleção, estão descritos na Tabela 1. 
Tabela 1. Resultados do processo de seleção de acordo com bases de dados.

\begin{tabular}{|c|c|c|c|c|c|c|}
\hline Base de dados & $\begin{array}{c}\text { Artigos } \\
\text { encontrados }\end{array}$ & $\begin{array}{l}\text { Excluídos por } \\
\text { repetição }\end{array}$ & $\begin{array}{l}\text { Seleção baseada } \\
\text { no título }\end{array}$ & $\begin{array}{c}\text { Seleção baseada } \\
\text { no resumo }\end{array}$ & $\begin{array}{c}\text { Seleção } \\
\text { baseada no } \\
\text { texto completo }\end{array}$ & $\begin{array}{c}\text { Artigos } \\
\text { selecionados (n) }\end{array}$ \\
\hline Science Direct & 32 & 2 & 1 & 1 & & \\
\hline Springer Science & 13 & 1 & 3 & 2 & 2 & 1 \\
\hline Medline/Pubmed & 24 & & 7 & 6 & 4 & 3 \\
\hline ProQuest LLC & 6 & 1 & 2 & 2 & 2 & 2 \\
\hline AGRIS & 2 & & & & & \\
\hline Bentham Science Publishers & 1 & & & & & \\
\hline Cengage Learning & 9 & 1 & 2 & 2 & 2 & 1 \\
\hline Web of Science & 1 & & & & & \\
\hline John Wiley \& Sons & 3 & & & & & \\
\hline Scopus & 2 & & & & & \\
\hline Directory of Open Access Journals & 3 & & 1 & 1 & & \\
\hline Maney Publishing & 1 & & & & & \\
\hline SAGE Publications & 1 & & & & & \\
\hline Total & & & & & & 98 \\
\hline Excluídos por repetição & & & & & & 5 \\
\hline Total da amostra (n) & 98 & 5 & 16 & 14 & 10 & 7 \\
\hline
\end{tabular}

Fonte: Autores.

Os estudos observacionais analíticos elencados avaliaram indivíduos de ambos os sexos, em diferentes faixas etárias, e basearam-se nos delineamentos tipo caso-controle $(n=3)$ e estudo transversal $(n=4)$, caracterizando toda a amostra com nível de evidência 4. Tabela 2 .

Tabela 2. Síntese dos estudos observacionais analíticos sobre as concentrações séricas de Zinco e suas relações com transtornos mentais comuns em humanos.

\begin{tabular}{|c|c|c|c|c|}
\hline Autor/ Ano & Grupo alvo & $\begin{array}{l}\text { Delineamento/ } \\
\text { Intervenção }\end{array}$ & $\begin{array}{l}\text { Método diagnósticos } \\
\text { dosagem do Zn/ TMC }\end{array}$ & Principais achados \\
\hline $\begin{array}{l}\text { Amani et al./ } \\
2010[25]\end{array}$ & $\begin{array}{l}\text { Amostra aleatória de } 308 \\
\text { estudantes, do sexo feminino, } \\
\text { de } 20-25 \text { anos de idade, foram } \\
\text { avaliados por escalas de } \\
\text { transtornos depressivos. } \\
\text { Destes, foram selecionados } 23 \\
\text { alunos com depressão } \\
\text { moderada a grave e } 23 \text { alunos, } \\
\text { da mesma idade, sem histórico } \\
\text { de depressão. }\end{array}$ & $\begin{array}{l}\text { Caso-controle } \\
\begin{array}{l}\text { Grupo 1: Depressão } \\
\text { moderada a grave }\end{array} \\
\begin{array}{l}\text { Grupo 2: Controle } \\
\text { (sem }\end{array} \\
\text { depressivos) }\end{array}$ & $\begin{array}{l}\text { Zn sérico: espectroscopia de } \\
\text { absorção atômica com } \\
\text { chama. Níveis séricos de } \mathrm{Zn} \\
\text { inferiores a } 70 \mu \mathrm{g} / \mathrm{dL} \text { foram } \\
\text { considerados como } \\
\text { deficientes. } \\
\text { Depressão: BDI* }\end{array}$ & $\begin{array}{l}\text { As concentrações séricas de } \mathrm{Zn} \text { do grupo com } \\
\text { depressão moderada a grave foram cerca de } \\
\text { dois terços do grupo controle. } \\
\text { Regressão linear negativa entre os scores do } \\
\text { BDI* e as concentrações séricas de zinco em } \\
\text { todos os indivíduos ( } \mathrm{r}=-0,65 ; \mathrm{p}<0,001 \text { ). }\end{array}$ \\
\hline $\begin{array}{l}\text { Islam et } \\
\text { al./2013 [26] }\end{array}$ & $\begin{array}{l}50 \text { pacientes com transtorno de } \\
\text { ansiedade generalizada e } 51 \\
\text { indivíduos saudáveis, ambos } \\
\text { os sexos, na faixa de } 18-55 \\
\text { anos de idade. }\end{array}$ & $\begin{array}{l}\text { Grupo1: Transtorno de } \\
\text { ansiedade generalizada } \\
\text { Grupo 2: Controle }\end{array}$ & $\begin{array}{l}\text { Zn sérico: espectroscopia de } \\
\text { absorção atômica com } \\
\text { chama. } \\
\text { Transtorno de ansiedade: } \\
\text { diagnóstico por psiquiatra } \\
\text { especialista treinado em } \\
\text { Manual Diagnóstico e } \\
\text { estatístico de Transtornos } \\
\text { psiquiátricos. }\end{array}$ & $\begin{array}{l}\text { As concentrações séricas de } \mathrm{Zn} \text { encontraram-se } \\
\text { significativamente reduzidas no grupo com } \\
\text { transtorno de ansiedade generalizada em } \\
\text { comparação ao grupo controle }(\mathrm{p}<0,05) \text {. }\end{array}$ \\
\hline
\end{tabular}


Markiewicz-

Zukowska,R;

Gutowsk,A; 100 participantes com idade de

Borawska,

60 a 102 anos
Zn sérico: espectroscopia de absorção atômica. Concentrações de $\mathrm{Zn}<0,7$ $\mathrm{mg} / \mathrm{L}$ foram consideradas deficientes.

Depressão: GDS**
Participantes com sinais de depressão apresentaram concentração sérica de $\mathrm{Zn}$ menor ( $\mathrm{p}=0,005)(0,77 \pm 0,17 \mathrm{mg} / \mathrm{L})$ do que aqueles sem depressão $(0,89 \pm 0,22 \mathrm{mg} / \mathrm{L})$. Não houve associação entre a concentração sérica de Zn e a intensidade da depressão( $\mathrm{p}=0,081)$.

$\mathrm{M} / 2015[12]$

Jung,A; Spira

D;

Steinhagem-

Thiessen,E;

Demuth,I;

Norman,

$\mathrm{K} / 2016[27]$
1514 idosos participantes do Study Aging Study II, sendo 722 mulheres. Idade entre 60 e 84 anos.
Transversal (análise transversal de amostra de estudo de coorte prospectivo sobre envelhecimento saudável).
Zn sérico: espectroscopia de absorção atômica com chama.

Sintomas depressivos: avaliados mediante "Center for Epidemiological Studies Depression" e GDS**.
Participantes com sintomas depressivos tiveram níveis mais baixos de $\mathrm{Zn}$ no plasma ( $\mathrm{p}=0,037$ ). Mesmo após o ajuste para preditores conhecidos de depressão, a deficiência de zinco no plasma permaneceu significativamente associada aos sintomas depressivos (odds ratio: 1,490 , intervalo de confiança de $95 \%$ : 1,027 $2,164 ; \mathrm{p}=0,036)$

\section{Caso-controle}

69 pacientes com quadro depressivo atual; 45 pacientes Styczeń et em remissão (idade média: al./2017 [28] $\quad 49,4 \pm 10,7)$; e 50 controles (idade média: 45,8 $\pm 12,4$ )
Grupo 1: Depressão

Grupo 2: Fase de remissão

Grupo 3: Controle
A concentração de $\mathrm{Zn}$ nas amostras de Zn sérico: espectroscopia pacientes na fase depressiva foi de absorção atômica com significativamente menor do que as obtidas no atomização eletrotérmica. grupo de voluntários saudáveis $(\mathrm{p}=0,003)$. No entanto, não houve diferenças significativas nos níveis de $\mathrm{Zn}$ entre pacientes que obtiveram remissão dos sintomas e o grupo controle $(\mathrm{p}=0,348)$.
Depressão: HDRS*** e MADRS****.
Zn sérico: espectroscopia de absorção atômica com
Correlação inversa significativa entre as concentrações séricas de zinco e os escores do $\mathrm{BDI}^{*}(\mathrm{r}=-0,23, \mathrm{p}<0,001)$.
100 alunas do ensino médio da 20 anos. chama.

Depressão: $\mathrm{BDI}^{*}$

Transversal

\section{8 meninas na faixa etária Transversal} entre 12-18 anos. Os participantes foram

Gonoodi et estratificados pelo seu escore 12018 [30] de depressão [338 (82,8\%) sujeitos com nenhum/mínimos sintomas e 70 indivíduos $(17,2 \%)$ com leves a graves sintomas de depressão].
(Grupamento e números aleatórios gerados por computador em diferentes áreas de Mashhad, nordeste do Irã)
Zn sérico: espectroscopia de absorção atômica com chama.

Depressão: $\mathrm{BDI}^{*}$
As concentrações séricas de zinco foram de $97.0 \pm 17,4 \mu \mathrm{g} / \mathrm{dL}$ e $95.3 \pm 17,8 \mu \mathrm{g} / \mathrm{dL}$, em indivíduos com nenhum sintoma (ou sintomas mínimos) de depressão ou sintomas leves a graves, respectivamente. Não houve correlação significativa nas concentrações séricas de zinco e o escore de depressão $(r=0,033, p=0,05)$.

Fonte: Autores. *BDI: Beck depression inventory; **GDS: Geriatric Depression Scale; *** HDRS: Hamilton Rating Scale for Depression; **** MADRS: Montgomery-Asberg Depression Rating Scale.

\section{Discussão}

Pesquisas da última década sugerem evidências conectando baixas concentrações séricas de zinco aos transtornos mentais comuns. Embora haja uma quantidade limitada de dados que relacionam este elemento à depressão e ansiedade, parece bastante razoável afirmar que o zinco se constitui em uma opção potencial na terapia psiquiátrica.

Todos os manuscritos elencados para análise (Markiewic-Żukowska et al., 2015; Amani et al., 2010; Islam et al., 2013; Jung et al., 2017; Styczeń et al., 2016; Tahmasebi et al., 2017; Gonoodi et al., 2018), conduzidos em grupos de diferentes faixas etárias e número amostral, apresentaram resultados semelhantes, demonstrando baixas prevalências nas concentrações de zinco no sangue (soro, plasma) de pacientes com depressão e/ou ansiedade. No entanto, em outros estudos, que investigaram a influência dos níveis de zinco sobre variadas enfermidades psiquiátricas e/ou os efeitos da suplementação de zinco em indivíduos depressivos, as concentrações de zinco no sangue não diferiram entre pacientes deprimidos e grupo de voluntários saudáveis de controle (Ranjbar et al., 2013; Gronli et al., 2013; Salustri, et al., 2011). 
No tocante à correlação entre concentrações séricas de zinco e transtornos mentais comuns, os resultados apresentados, à exceção dos achados de Gonoodi et al. (2018), convergem para evidências de que a insuficiência de zinco sérico está significativamente associada à sintomatologia depressiva e de ansiedade.

Similarmente, investigações de ensaios clínicos randomizados e controlados, conduzidas em grupos de diferentes faixas etárias e número amostral, apontam para evidências de que a suplementação de zinco está associada à redução de sintomas gerais e/ou internalizantes relacionados à depressão e ansiedade (Sawada \& Yokoi, 2010; DiGirolamo \& RamirezZea, 2010; Russo, 2011).

Possíveis divergências encontradas entre os resultados de investigações relativas à desregulação de zinco e TMC são comumente discutidas e podem originar-se de diferenças no tempo de medição do zinco, fase de depressão/remissão, diagnóstico de depressão (resistente ao tratamento/não resistente), farmacologia anterior/atual, duração da doença, gênero e consumo alimentar (Swardfager et al., 2013; Siwek et al., 2013; Nowak, 2015).

Baseando-se nos delineamentos das investigações elencadas, os mesmos não podem determinar uma relação de causalidade entre o zinco e os sintomas depressivos/ansiolíticos. Porém, é plausível afirmar, com bases nos resultados e mecanismos conhecidos da fisiologia do zinco, que a concentração periférica deste elemento pode desempenhar um papel na fisiopatologia de algum domínio da função mental (Rivas-García et al., 2018).

Consistente com os supostos efeitos da dosagem de zinco e causalidade de TMC, uma meta-análise (Swardfager et al., 2013) demonstrou que as concentrações de zinco foram aproximadamente $-1,85 \mu \mathrm{mol} / \mathrm{L}$ mais baixas em indivíduos deprimidos do que indivíduos de controle (intervalo de confiança de 95\%: [IC]: -2,51 a -1,19 $\mu \mathrm{mol} / \mathrm{L}, \mathrm{p}<0,00001$ ). Nesta investigação, os estudos que quantificaram os sintomas depressivos determinaram maior gravidade da depressão associada com maior deficiência relativa de zinco e os efeitos foram numericamente maiores em estudos de pacientes internados ( $\mathrm{p}<0,0001)$, quando comparados à amostras da comunidade $(\mathrm{p}=0,003)$.

DiGirolamo et al. (2010), ao concluírem que o aumento nas concentrações séricas de zinco foi associado às reduções nos sintomas internalizantes entre grupos suplementados com zinco e placebo, não observando diferenças significativas na melhoria da sintomatologia geral de depressão e ansiedade, pontuaram a possibilidade de que poucos meses podem não ser suficientes para que algumas das mudanças funcionais nos comportamentos e nos sintomas de saúde mental entrem em vigor (Vashum et al., 2014).

Salienta-se, ainda, a necessidade de considerar as interações de zinco com outros nutrientes neurotróficos importantes na plasticidade cerebral (Li et al., 2017; Nakamura et al., 2019). Estudo realizado em idosos australianos mostra, por exemplo, que maiores taxas da razão Cobre/Zinco foram associadas ao menor sofrimento psicológico. Porém, ao ser analisado isoladamente, o zinco sérico não foi associado ao desempenho cognitivo, sofrimento psicológico ou fatores neurotróficos (Mravunac et al., 2019).

Outra hipótese, relativa à suplementação, é de que o zinco seja mais eficaz em pacientes diagnosticados com condições de saúde mental mais graves e usado em conjunto com a farmacoterapia tradicional (Akhondzadeh et al., 2004), podendo atuar de forma coadjuvante e promissora no tratamento desses transtornos, atenuando os sintomas de depressão e ansiedade nesses indivíduos.

\section{Conclusão}

As investigações elencadas revelam uma complexidade importante às ações do zinco e apontam que os níveis séricos desse elemento se encontram reduzidos em grupos com depressão e ansiedade. Ademais, sugerem uma suplementação eficaz de zinco para que haja redução da sintomatologia psiquiátrica e, consequentemente, uma possível redução na quantidade de medicamentos psicotrópicos, levando à maior adesão ao tratamento, redução de custos e resultados mais favoráveis aos 
indivíduos em sofrimento mental.

Os resultados expostos despertam ainda o apoio do desenvolvimento e implementação de modelos de assistência multidisciplinar, visando a adequação dos níveis séricos de zinco. Neste sentido, realça-se a importância da ingestão de uma ampla variedade de alimentos fontes de zinco, independentemente da idade.

Além disso, faz-se necessária a realização de estudos mais refinados, objetivando elucidar o impacto do zinco nas condições neuropsiquiátricas, visto que certos subconjuntos parecem ser mais afetados com a desregulação deste elemento.

\section{Referências}

Akhondzadeh, S., Mohammadi, M. R., \& Khademi, M. (2004). Zinc sulfate as an adjunct to methylphenidate for the treatment of attention deficit hyperactivity disorder in children: a double blind and randomized trial [ISRCTN64132371]. BMC Psychiatry, 4 (9), 1-6. https://bmcpsychiatry.biomedcentral.com/articles/10.1186/1471-244X-4-9.

Amani, R., Saeidi, S., Nazari, Z., \& Nematpour, S. (2010). Correlation Between Dietary Zinc Intakes and Its Serum Levels with Depression Scales in Young Female Students. Biol Trace Elem Res, 137 (2), 150-158. https://link.springer.com/article/10.1007/s12011-009-8572-x.

Campo, M. T., Ramon, E., Lupala, C. S., Pérez, J. J., Koch, K., \& Garriga, P. (2016). Zinc Is Involved in Depression by Modulating G Protein-Coupled Receptor Heterodimerization. Mol Neurobiol, 53 (3), 2003-2015. https://pubmed.ncbi.nlm.nih.gov/25855059/.

Ciampo, L. A. D., \& Ciampo, L. R. S. (2014). The importance of zinc for adolescent health. Adolesc. Saúde, 11 (2), 80-86. https://cdn.publisher.gn1.link/adolescenciaesaude.com/pdf/v11n2a11.pdf.

DiGirolamo, A. M., \& Ramirez-Zea, M. (2010). Role of zinc in maternal and child mental health. Am J Clin Nutr, 89 (suppl), 940S-945S. https://academic.oup.com/ajcn/article/89/3/940S/4596790.

Doboszewska, U., Wlaź, P., Nowak, G., Radziwoń-Zaleska, M., Cui, R., \& Młyniec, K. (2017). Zinc in the monoaminergic theory of depression: its relationship to neural plasticity. Neural Plast, 1-18. https://www.hindawi.com/journals/np/2017/3682752/.

Gonoodi, K., Moslem, A., Ahmadnezhad, M., Darroudi, S., Mazloum, Z., Tayefi, M., Tabatabaeizadeh, S. A., Eslami, S., Shafiee, M., Khashayarmanesh, Z., Haghighi, H. M., Ferns, G. A., \& Ghayour-Mobarhan, M. (2018). Relationship of Dietary and Serum Zinc with Depression Score in Iranian Adolescent Girls. Biological Trace Element Research, 186 (2), 1-7. https://link.springer.com/article/10.1007\%2Fs12011-018-1301-6.

Gronli, O., Kvamme, J. M., Friborg, O., \& Wynn, R. (2013). Zinc deficiency is common in several psychiatric disorders. PLoS One, 8 (12). https://www.ncbi.nlm.nih.gov/pmc/articles/PMC3868572/.

Henriques, R. T. M., Cabana, M. C. F. L., \& Montarroyos, U. R. (2018). Prevalence of Common Mental Disorders associated with the burden placed on family caregivers of elderly. Mental, $12 \quad$ (22), 35-52. http://pepsic.bvsalud.org/scielo.php?script=sci_abstract\&pid=S1679$44272018000100004 \& \operatorname{lng}=\mathrm{pt} \& n r m=\mathrm{iso} \& \operatorname{lng}=\mathrm{en}$.

Islam, M. R., Ahmed, M. U., Mitu, S. A., Islam, M. S., Rahman, G. K. M. M., Qusar, M. M. A. S., \& Hasnat, A. (2013). Comparative Analysis of Serum Zinc, Copper, Manganese, Iron, Calcium, and Magnesium Level and Complexity of Interelement Relations in Generalized Anxiety Disorder Patients. Biol Trace Elem Res, 154 (1), 21-27. https://link.springer.com/article/10.1007\%2Fs12011-013-9723-7.

Joe, P., Getz, M., Redman, S., Petrilli, M., Kranz, T. M., Ahmad, S., \& Malaspina, D. (2018). Serum zinc levels in acute psychiatric patients: A case series. Psychiatry Research, 261: 344-350. https://www.sciencedirect.com/science/article/abs/pii/S0165178117313070.

Jung, A., Spira, D., Steinhagen-Thiessen, E., Demuth, I., \& Norman, K. (2017). Zinc Deficiency Is associated With Depressive Symptoms-Results from the Berlin Aging Study II. J Gerontol A Biol Sci Med Sci, 72 (8), 1149-1154. https://academic.oup.com/biomedgerontology/article/72/8/1149/2328605.

Kaner, G., Soylu, M., Yüksel, N., Inanç, N., Ongan, D., Başmısırlı, E. (2015). Evaluation of Nutritional Status of Patients with Depression. BioMed Res Int, 19. https://pubmed.ncbi.nlm.nih.gov/26413529/.

Li, Z., Li, B., Song, X., \& Zhang, D. (2017). Dietary zinc and iron intake and risk of depression: A meta-analysis. Psychiatry Res, 251, 41-47. https://www.sciencedirect.com/science/article/abs/pii/S0165178116317747?via\%3Dihub.

Li, Z., Wang, W., Xin, X., Song, X., \& Zhang, D. (2018). Association of total zinc, iron, copper and selenium intakes with depression in the US adults. Journal of Affective Disorders. 228, 68-74. https://pubmed.ncbi.nlm.nih.gov/29232566/.

Manosso, L. M. (2016). Investigação de mecanismos de ação envolvidos no efeito tipo-antidepressivo do zinco em camundongos [tese] [internet]. [Florianópolis]: Universidade Federal de Santa Catarina, 254 p. https://repositorio.ufsc.br/handle/123456789/167962.

Manosso, L. M. (2019). O papel do zinco no transtorno depressivo maior. Rev Bras Nut Func, 43 (78), 1-19. https://www.researchgate.net/publication/334177606_O_papel_do_zinco_no_transtorno_depressivo_maior.

Markiewic-Żukowska, R., Gutowska, A., \& Borawska, M. H. (2015). Serum Zinc Concentrations Correlate with Mental and Physical Status of Nursing Home Residents. PLoS One, 10 (1). https://www.ncbi.nlm.nih.gov/pmc/articles/PMC4311908/.

Mravunac, M., Szymlek-Gay, E. A., Daly, R. M., Roberts, B. R., Formica, M., Gianoudis, J., O’Connell, S. L., Nowson, C. A., \& Cardoso, B. R. (2019). Greater Circulating Copper Concentrations and Copper/Zinc Ratios are Associated with Lower Psychological Distress, But Not Cognitive Performance, in a Sample of Australian Older Adults. Nutrients, 11(10). https://www.mdpi.com/2072-6643/11/10/2503. 
Młyniec, K., Gaweł, M., \& Nowak, G. (2015). Study of antidepressant drugs in GPR39 (zinc receptor-/-) knockout mice, showing no effect of conventional antidepressants, but effectiveness of NMDA antagonists. Behav. Brain Res, 287, 135-138. https://www.sciencedirect.com/science/article/abs/pii/S0166432815002181?via\%3Dihub.

Mlyniec, K. (2015). Zinc in the Glutamatergic Theory of Depression. Current Neuropharmacology, 13 (4), 505-513. https://www.ncbi.nlm.nih.gov/pmc/articles/PMC4790399/\#: :text=Based\%20on\%20glutamatergic\%20theory\%20of,inhibition\%20of\%20the\%20NMDA\%20r eceptor.

Nakamura, M., Miura, A., Nagahata, T., Shibata, Y., Okada, E., \& Ojima, T. (2019). Low Zinc, Copper, and Manganese Intake is Associated with Depression and Anxiety Symptoms in the Japanese Working Population: Findings from the Eating Habit and Well-Being Study. Nutrients, 11(4). https://www.mdpi.com/2072-6643/11/4/847\#.

Nguyen, T. T. T., Miyagi, S., Tsujiguchi, H., Kambayashi, Y., Hara, A., Nakamura, H., Suzuki, K., Yamada, Y., Shimizu, Y., \& Nakamura, H. (2019). Association between Lower Intake of Minerals and Depressive Symptoms among Elderly Japanese Women but Not Men: Findings from Shika Study. Nutrients, 11 (2). https://pubmed.ncbi.nlm.nih.gov/30781841/.

Nowak, G. (2015). Zinc, future mono/adjunctive therapy for depression: mechanisms of antidepressant action. Pharmacol Rep, 67 (3), 659-662. https://link.springer.com/article/10.1016/j.pharep.2015.01.015.

Petrilli, M. A., Kranz, T. M., Kleinhaus, K., Joe, P., Getz, M., Johnson, P., Chao, M. V., \& Malaspina, D. (2017). The Emerging Role for Zinc in Depression and Psychosis. Front Pharmacol, 30 (8). https://www.frontiersin.org/articles/10.3389/fphar.2017.00414/full.

Ranjbar, E., Kaseaei, M. S., Mohammad-Shirazi, M., Nasrollahzadeh, J., Rashidkhani, B., Shams, J., Mostafari, S. A., \& Mohammadi, M. R. (2013). Effects of zinc supplementation in patients with major depression, a randomized clinical trial. J Psychiatry, 8, 73-79. https://www.ncbi.nlm.nih.gov/pmc/articles/PMC3796297/.

Rivas-García, T. E., Marcelo-Pons, M., Martínez-Arnau, F., Serra-Catalá, N., Santamaría-Carrillo, Y., \& Cauli, O. (2018). Blood zinc levels and cognitive and functional evaluation in non-demented older patients. Exp Gerontol, 108, 28-34. https://www.sciencedirect.com/science/article/abs/pii/S0531556518300639?via\%3Dihub.

Russo, A. J. (2011). Decreased Zinc and Increased copper in Individuals with Anxiety. Nutr Metab Insights, 4, 1-5. https://journals.sagepub.com/doi/10.4137/NMI.S6349.

Salustri, C., Squitti, R., Zappasodi, F., Ventriglia, M., Bevacqua, M. G., Fontana, M., \& Tecchio, F. (2011). Oxidative stress and brain glutamate-mediated excitability in depressed patients. J Affect Disord, 127, 321-325. https://www.sciencedirect.com/science/article/abs/pii/S0165032710003976.

Santos, G. B. V., Alves, M. C. G. P., Goldbaum, M., Cesar, C. L. G., \& Gianini, R. J. (2019). Prevalência de transtornos mentais comuns e fatores associados em moradores da área urbana de São Paulo, Brasil. Cad. Saúde Pública, 35 (11). https://www.scielo.br/scielo.php?pid=S0102311X2019001305008\&script=sci_abstract.

Saueressig, C., Silva, V. L., Antunes, L. C., Alba, V. D. (2016). Níveis de zinco sérico em pacientes internados com depressão. J. Bras. Psiquiatr, 65 (3), 239 $244 . \quad$ https://www.scielo.br/scielo.php?script=sci_arttext\&pid=S0047-20852016000300239\#: :text=Os\%20dados\%20da\%20literat ura $\% 20$ quanto,dentro\%20da $\% 20$ normalidade $17 \% 2 \mathrm{C} 18$.

Sawada, T., \& Yokoi, K. (2010). Effect of zinc supplementation on mood states in young women: a pilot study. European Journal of Clinical Nutrition, 64 (3), 331-333. https://www.nature.com/articles/ejen2009158.

Swardfager, W., Herrmann, N., Mazereeuw, G., Goldberger, K., Harimito, T., \& Lanctôt, K. L. (2013). Zinc in depression: a meta-analysis. Biol. Psychiatry, 74, 872-878. https://www.biologicalpsychiatryjournal.com/article/S0006-3223(13)00451-4/fulltext.

Senicato, C., Azevedo, R. C. S., \& Barros, M. B. A. (2018). Common mental disorders in adult women: identifying the most vulnerable segments. Ciênc. Saúde coletiva, 23 (8). https://doi.org/10.1590/1413-81232018238.13652016.

Silva, A. C., Vargas, L. S., Moraes, R. C. C., Lucchese, R., Guimarães, R. A., \& Vera, I. (2019). Prevalência e fatores associados ao transtorno mental comum em assentados rurais*. SMAD, Rev. Eletrônica Saúde Mental Álcool Drog, 15(1), 23-31. http://dx.doi.org/10.11606/issn.1806-6976.smad.2019.00037.

Siwek, M., Szewczyk, B., Dudek, D., Styczeñ, K., Sowa-Kuæma, M., Mlyniec, K., Siwek, A., Witkowski, L., Pochwat, B., \& Nowak, G. (2013). Zinc as a marker of affective disorders. Pharmacol Rep, 65, 1512-1518. https://www.sciencedirect.com/science/article/abs/pii/S1734114013715123.

Souza, M, T., Silva, M. D., \& Carvalho, R. C (2010). Revisão integrativa: o que é e como fazer. Einstei, 8 (1), 102-106. https://www.scielo.br/scielo.php?pid=S1679-45082010000100102\&script=sci_arttext\&tlng=pt.

Styczeń, K., Sowa-Kućma, M., Siwek, M., Dudek, D., Reczyński, W., Szewczyk, B., Misztak, P., Topór-Madry, R., \& Nowak, G. (2016). The serum zinc concentration as a potential biological marker in patients with major depressive disorder. Metab Brain Dis, 32, 97-103. https://link.springer.com/article/10.1007\%2Fs11011-016-9888-9.

Tahmasebi, K., Amani, R., Nazari, Z., Ahmadi, K., Moazzen, S., \& Mostafavi, S.-A. (2017). Association of Mood Disorders with Serum Zinc Concentrations in Adolescent Female Students. Biol Trace Elem Res, 178 (2), 180-188. https://doi.org/10.1007/s12011-016-0917-7.

Ursi, E. S (2005). Prevenção de lesões de pele no perioperatório: revisão integrativa da literatura. Universidade de São Paulo; 129 p. https://www.teses.usp.br/teses/disponiveis/22/22132/tde-18072005-095456/pt-br.php.

Vashum, K. P., McEvoy, M., Milton, A. H., McElduff, P., Hure, A., Byles, J., \& Attia, J. (2014). Dietary zinc is associated with a lower incidence of depression: findings from two Australian. Journal of Affective Disorders, 166, 249-257. https://www.sciencedirect.com/journal/journal-of-affectivedisorders/vol/166/suppl/C. 
Research, Society and Development, v. 10, n. 6, e3410615441, 2021

(CC BY 4.0) | ISSN 2525-3409 | DOI: http://dx.doi.org/10.33448/rsd-v10i6.15441

Youssef, M. M., Underwood, M. D., Huang, Y. Y., Hsiung, S. C., Liu, Y., Simpson, N. R., Bakalian, M. J., Rosoklija, G. B., Dwork, A. J., Arango, V., \& Mann, J. J. (2018). Association of BDNF Val66Met Polymorphism and Brain BDNF Levels with Major Depression and Suicide. Int J Neuropsychopharmacol, 21 (6), 528-538. https://academic.oup.com/ijnp/article/21/6/528/4843985.

World Health Organization [WHO] (2015). Compreensão do plano de ação de saúde mental para 2013-2020-2030. Geneva: World Health Organization; 2015. http://www.who.int/mental_health/action_plan_2013/en/

World Health Organization [WHO] (2017). Depression and Other Common Mental Disorders: Global Health Estimates. Geneva: World Health Organization; 2017. https://apps.who.int/iris/handle/10665/254610.

World Health Organization [WHO] (2020a). Fact sheet $n^{o}$ 369: depression. Geneva: World Health Organization; http://www.who.int/mediacentre/factsheets/fs369/en/.

World Health Organization [WHO] (2020b). Improving the mental and brain health of children and adolescentes. Child and adolescent mental health. https://www.who.int/mental_health/maternal-child/child_adolescent/en/.

World Health Organization [WHO], Organização Pan-Americana da Saúde [OPAS] (2020). Folha Informativa. Transtornos Mentais. https://www.paho.org/pt/topicos/transtornos-mentais. 